\title{
All-Optical 160 Gbit/s Retiming System Using Fiber Grating Based Pulse Shaping Technology
}

\author{
F. Parmigiani (1), L.K. Oxenløwe (2), M. Galili (2), M. Ibsen (1), D. Zibar (2), P. Petropoulos (1), D.J. \\ Richardson (1), A.T. Clausen (2) and P. Jeppesen (2)
}

\begin{abstract}
This paper demonstrates a retiming system operating at rates of 40 and $160 \mathrm{Gbit} / \mathrm{s}$, which incorporates a superstructured fiber Bragg grating (SSFBG) as a pulse shaping element. The original data pulses are shaped into flattopped (rectangular) pulses to avoid conversion of their timing jitter into pulse amplitude noise at the output of a nonlinear fiber-based Kerr switch. Thus retiming is performed in a single step avoiding wavelength conversion. The benefits of using shaped rather than conventional pulse forms in terms of timing jitter reduction are confirmed by bit-error rate (BER) measurements.
\end{abstract}

Index Terms-All-optical regeneration, timing jitter, fiber Bragg grating, nonlinear switches, optical pulse shaping, ultrashort optical pulses.

\section{INTRODUCTION}

A $\mathrm{s}$ data rates increase, and the associated pulse durations get shorter, it becomes critical to be able to manage the noise-related transmission impairments on the original data pulses, such as amplitude and timing jitter. At bit rates beyond $40 \mathrm{Gbit} / \mathrm{s}$, it is considered both technically (due to the intrinsic benefits in speed) and economically (due to the generally lower power consumption requirements) beneficial to consider all-optical techniques for the mitigation of such impairments. Nevertheless, the issue of bit retiming is particularly challenging, since, apart from clock recovery, it also requires the availability of a switch with a uniform response over a time base extending across the duration of the bit slot. Whereas this might be conceptually straightforward in digital electronics, the analogue nature of nonlinear optical devices, whose response depends directly on the intensity of the control signal, introduces an extra architectural complexity.

Manuscript received April, 2008. This work was supported in part by by the Danish Research Council (project Ultra-Net), the EU (COST-291 and NoE ePhoton/ONe+) and the STREP TRIUMPH (IST-027638). M. Ibsen acknowledges the support from a Royal Society University Research Fellowship.

F. Parmigiani, M. Ibsen, P. Petropoulos and D.J. Richardson are with the Optoelectronics Research Centre, University of Southampton, SO17 1BJ, United Kingdom; e-mail: frp@orc.soton.ac.uk).

L.K. Oxenløwe, M. Galili, D. Zibar, A.T. Clausen and P. Jeppesen are with COM-DTU, Technical University of Denmark, Bld. 343, DK-2800 Kgs. Lyngby, Denmark (e-mail: lo@com.dtu.dk).
One route towards achieving an extended switching window is by shaping the control optical pulse at the switch input into a rectangular waveform [1-3]. This provides then optimal resilience to timing jitter induced errors, and also reduces the absolute accuracy for temporal bit alignment. The shaping and manipulation of the optical pulses are conventionally achieved using liquid crystal spatial modulators [4-5] or arrayed-waveguide gratings (AWGs) [6]. In literature, picosecond and sub-picosecond long rectangular switching windows have been also demonstrated using uniform long periodic gratings operating in transmission [78] and propagation in a section of polarisation maintaining fibre [3]. In this last scheme, the pulse shaper is based on obtaining controllable pulse broadening in a $\mathrm{Hi}-\mathrm{Bi}$ fibre due to polarisation mode walk-off.

In this paper, we demonstrate timing jitter reduction in an optical time division multiplexing (OTDM) system operating at data rates up to $160 \mathrm{Gbit} / \mathrm{s}$ by linearly reshaping RZ data pulses in a polarization insensitive SSFBG into longer rectangular (flat-top) pulses ( $\sim 5 \mathrm{ps})$ and then switching these pulses in a nonlinear Kerr switch with a synchronous optical clock signal [3, 9-10], while maintaining the original wavelength. SSFBG technology offers a ready route to the production of optical filters with very precisely controlled frequency and phase profiles of almost arbitrary complexity in a single continuous grating structure and which can therefore be used to manipulate the properties of incident optical signals. An SSFBG is defined as a standard fibre grating, i.e. a fiber with a rapidly varying refractive index modulation of uniform amplitude and pitch, onto which an additional, slowly varying amplitude/phase modulation has been imposed along its length [11]. In the low grating reflectivity limit, i.e. for which the light penetrates the full grating length, the optical frequency response of the grating is simply given by the Fourier transform of its spatial refractive index modulation profile, thereby allowing the spatial information of the grating superstructure to be directly mapped into the time domain [12].

SSFBGs have proven to be an extremely powerful tool for advanced photonic applications that require fine and accurate manipulation of the shape of short optical pulses, thanks to the high precision in the implementation of the superstructure phase and amplitude profiles offered by plane-by-plane 
grating fabrication techniques. Using this linear all-fiberized pulse shaping method, we have previously demonstrated the reshaping of short pulses of a few picoseconds duration into rectangular pulses of different pulse widths (10-20ps) [10, 1214]. However, this extremely versatile technique is suitable for a far broader range of applications, ranging from optical code generation/recognition $[15,16]$, repetition rate multiplication [17], data format conversion [18], regeneration [19] and compression [20], to more exotic applications, such as Optical Fourier Transformation (OFT) [21].

This paper is organized as follows. In Section II, we present the principle of operation of the scheme and the pulse characterisation of the $\sim 5 \mathrm{ps}$ rectangular pulses. Section III describes the experimental set-up we used, while the performance of the proposed scheme as a pure retimer is assessed in Section IV. In this Section, we show detailed timing jitter characterisations and BER measurements. These results were obtained at data rates of both 40 and $160 \mathrm{Gbit} / \mathrm{s}$. Comparative measurements are also presented when shaping the data signal into Gaussian pulses with similar pulse durations as the rectangular pulses, highlighting the improvement obtained with the pulse shaping grating.

\section{PRINCIPLE OF OPERATION AND PULSE CHARACTERISATION}

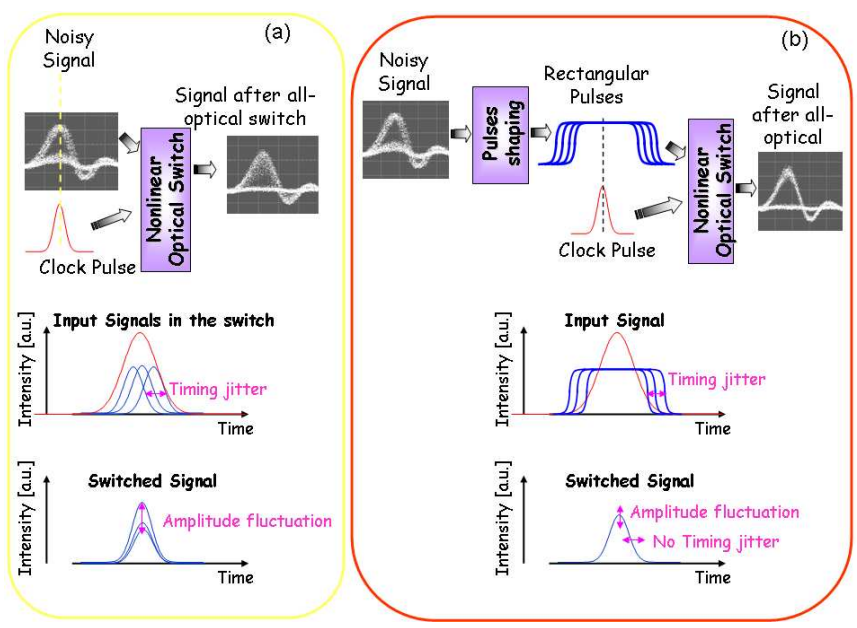

Figure 1. Operating principle of the retiming technique without $(a)$ and with $(b)$ the use of a rectangular pulse shape.

The basic idea behind the retiming scheme is shown in Fig. 1. The Figure illustrates the effect of timing jitter on the performance of an optical switch (controlled by a short optical clock pulse) in the instance that: (a) no retiming; or (b) retiming in the form of pulse reshaping in the form of a rectangular waveform is applied to the signal. As can be appreciated in case (a), large timing jitter in the signal pulses results in significant pulse amplitude noise at the output of the switch when the signal is switched by a short conventional pulse shape. However, if the incoming data signal is shaped into a rectangular pulse prior to being injected into the switch (case (b) in Fig.1), the flat top of the new shape ensures that the switch is insensitive to coarse mistiming within the original

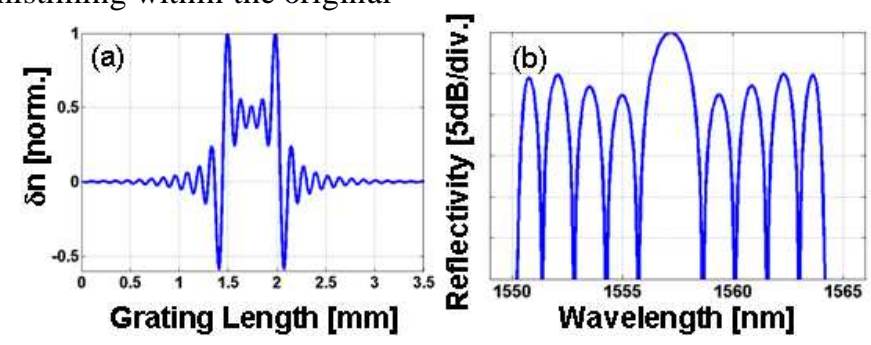

Figure 2. Refractive index modulation profile of the SSFBG (a) and corresponding simulated spectral response $(b)$.
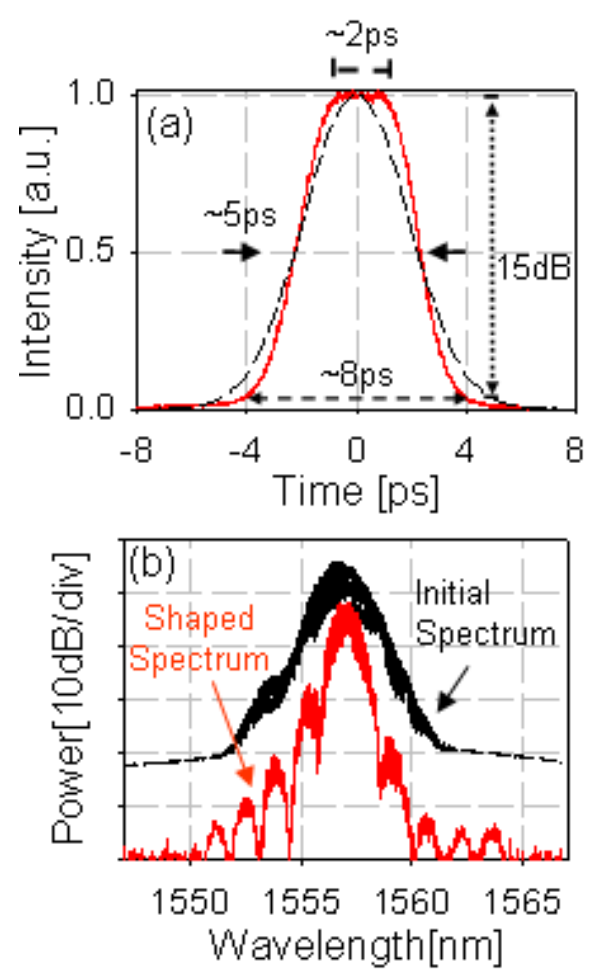

Figure 3. a) Cross-correlation measurement of the shaped 5 ps rectangular pulse (solid line) and its comparison with a Gaussian pulse shape (dashed line) with a similar FWHM $(\sim 5 p s) . b)$ Spectral traces before and after the SSFBG.

signal. The maximum tolerable amount of timing jitter that can be suppressed is defined by the duration of the rectangular pulse and cannot be larger than the duration allocated to each data slot. The signal can then be switched by clean, short clock signal pulses that are temporally aligned to the nominal center of the rectangular data pulses to ensure that the output signal is properly retimed.

In our experiments, we generate $\sim 5 \mathrm{ps}$ rectangular pulses using our SSFBG based pulse-shaping technology. As we already discussed in the previous Section, this is a purely passive-filtering process; to be able to properly design the 
grating and, thus, achieve the desired pulse shape, the characteristics of the pulses which are to be fed to the grating input need to be known in advance. The SSFBG has been designed and fabricated with the correct phase and amplitude reflectivity profile to work optimally with a transform-limited $\sim 1$ ps Gaussian pulse at its input. We chose this particular pulse duration to ensure that we could accommodate an adequate number of spectral features of the SSFBG reflectivity within the available spectral bandwidth of the incoming signal, while avoiding to overcomplicate the SSFBG refractive index modulation profile. The corresponding simulated refractive index modulation profile and spectral response are shown in Fig. 2(a) and Fig. 2(b). The peak reflectivity of the grating is $\sim 20 \%$, corresponding to a maximum modulation index of $\delta \mathrm{n} \sim 10^{-3}$. We can accommodate 9 spectral lobes within the available $\sim 14 \mathrm{~nm}$ spectral bandwidth, while the main body of the grating is written within $\sim 2.5 \mathrm{~mm}$ of fibre, emphasizing the high precision required during the fabrication process. Note that in this demonstration however, we used slightly broader pulses $(\sim 1.8 \mathrm{ps})$, so that the shaped pulses have smoother edges and a narrower flat top than those targeted [12]. Fig. 3(a) shows the corresponding temporal profile of the pulse shaping grating, measured with a cross-correlator and using a $\sim 600$ fs sampling pulse. The flat top of the shaped pulse extends over $\sim 2$ ps. As a reference, we also include in the same figure a plot of a Gaussian pulse with a similar full width at half maximum (FWHM).

Fig. 3(b) compares the spectra both before and after the filtering, where the shaping function of the grating and the appearance of the sinc-like features corresponding to a rectangular waveform can be clearly observed. The SSFBG response is broader than the input spectrum, as previously explained, while the spectral asymmetry observed results from the slight spectral response asymmetry of the SSFBG.

\section{EXPERIMENTAL SET-UP}

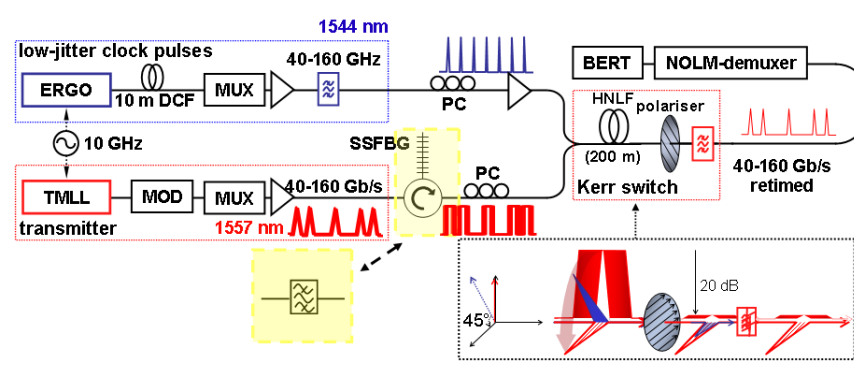

Figure 4. Retiming set-up at 40- and 160-Gbit/s. Inset: cartoon showing the operating principle of the Kerr switch.

The experimental set-up is reported in Fig. 4. A tunable mode-locked laser (TMLL) operating at $\sim 1557 \mathrm{~nm}$ is used to generate $\sim 1.8 \mathrm{ps}$ Gaussian pulses at a repetition rate of 10 GHz. The laser source has an inherent RMS timing jitter of $\tau_{\mathrm{rms}} \sim 420$ fs. These pulses are then amplitude modulated to provide a $2^{7}-1$ pseudorandom bit sequence (PRBS) and subsequently multiplexed up to either 40 or $160 \mathrm{Gbit} / \mathrm{s}$ according to the specific experiment. The data signal is shaped via the SSFBG into $\sim 5$ ps rectangular pulses and fed into the Kerr switch. The nonlinear element in the switch is constituted by $\sim 200 \mathrm{~m}$ of highly nonlinear fiber (HNLF) with a nonlinear coefficient $\gamma \sim 10.5 \mathrm{~W}^{-1} \mathrm{~km}^{-1}$, a dispersion slope and a

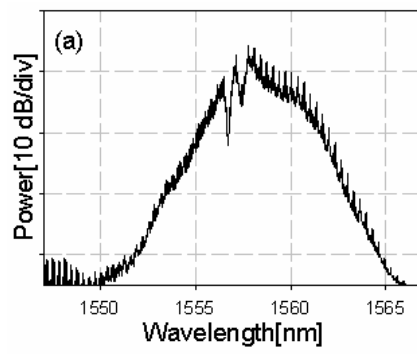
zero dispersion

Figure 5. Spectra after the Kerr switch at 40Gbit/s (a) and $160 \mathrm{Gbit} / \mathrm{s}(\mathrm{b})$.

wavelength of $0.018 \mathrm{ps} / \mathrm{nm}^{2} / \mathrm{km}$ and $\sim 1554 \mathrm{~nm}$, respectively.

The clock source, synchronised to the data stream via the same RF synthesizer, is an Erbium-glass mode-locked laser (ERGO) with low inherent timing jitter $\left(\tau_{\mathrm{rms}} \sim 210 \mathrm{fs}\right)$, operating at $1544 \mathrm{~nm}$ and providing pulses with a FWHM of 1.3 ps when properly compressed in a short length of dispersion compensating fiber. These pulses are multiplexed up to either 40 or $160 \mathrm{GHz}$ and amplified before being launched as the control signal onto the Kerr switch.

The data signal is aligned at $90^{\circ}$ to the Kerr switch polariser so as to be attenuated in the Kerr switch when the control signal is absent. The clock pulses co-propagating in the Kerr switch are aligned at $45^{\circ}$ relative to the shaped data pulses. The part of the square pulse that overlaps in time with the clock pulse has its polarisation rotated within the HNLF due to cross-phase modulation and can thus be transmitted through the polariser (see inset of Fig.4). Since the walk-off between the two signals inside the HNLF is negligible owing to the low fibre dispersion, the data pulses after the retiming scheme retain a similar pulse width (FWHM 1.2ps) and RMS timing jitter ( $\tau_{\mathrm{rms}} \sim 250 \mathrm{fs}$ ) as the control signal.

The retimed signal is then characterized using the corresponding diagnostics and subsequently demultiplexed down to $10 \mathrm{Gbit} / \mathrm{s}$. This was obtained using a non-linear optical loop mirror (NOLM), which was controlled by a $10 \mathrm{GHz}$ clean synchronized signal, with $50 \mathrm{~m}$ HNLF (slope $0.018 \mathrm{ps} / \mathrm{nm}^{2} \mathrm{~km}$, zero dispersion wavelength at $1554 \mathrm{~nm}$, $\gamma \sim 10.5 \mathrm{~W}^{-1} \mathrm{~km}^{-1}$ ) as a demultiplexer before entering the BER tester (BERT).

Fig. 5(a) and Fig. 5(b) show the spectra at the output of the retiming scheme for operation at 40 and $160 \mathrm{Gbit} / \mathrm{s}$ respectively, highlighting that the spectra lose their sincshape when properly switched and broaden with respect to the initial pulse spectrum (see Fig. 3(b)) while still centred on the 
original wavelength. The two spectra clearly show the $40 \mathrm{GHz}$ and $160 \mathrm{GHz}$ tones, while the specific feature, which is common between the two traces, due to the similar peak power considered, is due to nonlinearity (mainly self-phase modulation and XPM) in the HNLF.

To study the benefit of the rectangular switching window compared to more conventional shapes, the SSFBG was then replaced by a conventional narrow Gaussian filter that shapes the incoming signal into Gaussian pulses of similar shape as the rectangular pulses, see Fig. 3(a).

\section{PULSE RETIMING: EXPERIMENTAL RESULTS}

In order to evaluate the performance of the proposed system, we started by examining the retiming properties at 40Gbit/s, since this data rate allows us to measure the RMS timing jitter at the FWHM point on the trailing edge of the pulse on a Digital Communication Analyser (DCA) using an Agilent Precision Timebase Module. The results are reported in Table 1 and clearly show that the pulses at the output of the Kerr switch have their jitter drastically reduced from $\sim 420$ fs to $\sim 250 \mathrm{fs}$ (close to the clock pulse jitter of $\sim 210 \mathrm{fs}$ ), albeit with a slight increase in amplitude noise. The corresponding amplitude noise standard deviation values for the marks $\left(\sigma_{\text {a.j. }}\right)$, which are normalized to their mean values, are also reported in Table 1. The increase in the switched pulse amplitude noise may be linked to the non-uniformity of the rectangular pulse shape, to slight spectral distortions of the rectangular pulses caused by self-phase modulation experienced during propagation in the HNLF and to the inline amplifiers used. This indicates than ideally an amplitude regeneration stage should also be incorporated within the system.

In order to confirm the necessity for a retiming system we first compare the performance of the system when the retiming stage is applied either through the use of the pulse shaping SSFBG or through a conventional Gaussian shape, to that when no retiming is applied at all (Fig.6).

We started by characterising the switched data signal at 40Gbit/s when no pulse shaping was applied. An error floor at a BER $\sim 10^{-7}$ was encountered for the maximum receiver power of $\sim-20 \mathrm{dBm}$. In contrast, with the SSFBG in place, error-free operation was achieved for all 4 retimed channels and the corresponding opened eye diagrams are reported in Fig. 7(a). We then replaced the flat-top pulses with $\sim 5 \mathrm{ps}$ Gaussian pulses (see Fig. 3(a)) and repeated the same measurements. At $40 \mathrm{Gbit} / \mathrm{s}$, error free operation was achieved with only a very small power penalty compared to the flat top pulse shape and the corresponding eye diagrams are reported in Fig. 7(b). These results reflect the relatively low amount of RMS timing jitter associated with the data signal. The RMS timing jitter associated to the data signal and the two pulse shapes (flat-top and Gaussian) are sketched in Fig. 8(a). If the data pulses exhibited a larger amount of timing jitter then a severe BER degradation would be evident in the measurements for Gaussian pulse shaped data signal.

\begin{tabular}{|c|c|c|c|}
\hline & Original pulse & $\begin{array}{c}\text { Clock } \\
\text { pulse }\end{array}$ & Retimed pulse \\
\hline$\tau_{\text {rms }}[\mathrm{fs}]$ & 420 & 210 & 250 \\
\hline$\sigma_{\text {a.j. }}[\%]$ & 3.6 & 2.4 & 7.1 \\
\hline
\end{tabular}

Table 1: Timing and amplitude jitter values at different points of the system for operation at 40Gbit/s.
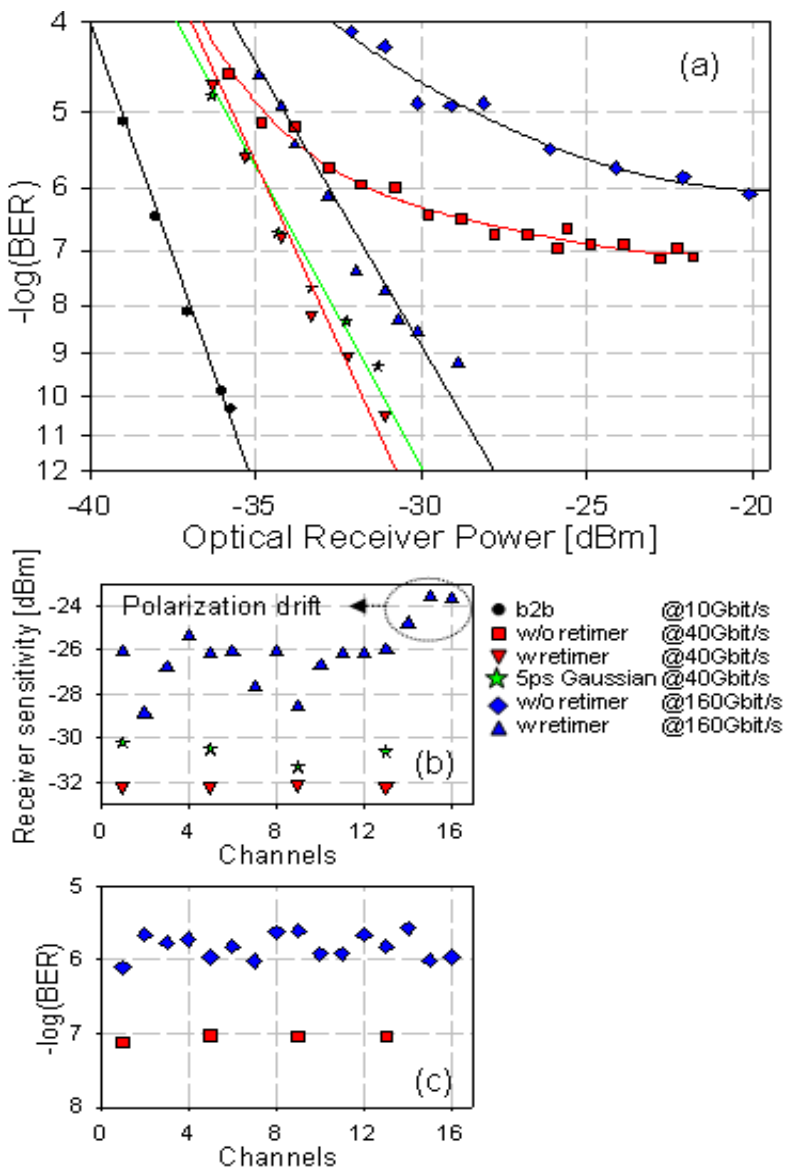

Figure 6. a) BER characteristics of all channels at $40 \mathrm{Gbit} / \mathrm{s}$ and $160 \mathrm{Gbit/s}$ with and without any retiming scheme. b) Receiver sensitivity for all channels at $40 \mathrm{Gbit} / \mathrm{s}$ when the data pulses are rectangularly shaped (triangles down) and Gaussian pulses of a similar pulse width (stars). The same measurements at 160Gbit/s for shaping into rectangular pulses only (triangles up). c) BER at 40Gbit/s and 160Gbit/s without any retiming scheme at the maximum receiver power of $\sim-20 \mathrm{dBm}$

We then increased the bit rate of the signal to $160 \mathrm{Gbit} / \mathrm{s}$ and performed similar assessments. Once again, without retiming, an error floor at $\mathrm{BER} \sim 10^{-6}$ was obtained for the maximum receiver power, as shown in Fig. 6(a). When flattopped pulses were used, error-free operation for all 16 retimed channels was achieved. The spread in sensitivity 
among the retimed channels is due to uneven amplitudes in the multiplexed pulses in the data and clock arms, and to polarisation drifts in the Kerr switch (see last three channels in Fig. 6(b)). The corresponding switched eye diagrams are reported in Fig. 7(c).

We then used the $\sim 5$ ps Gaussian pulses and complete closure of the eyes after the Kerr switch was observed (as shown in Fig. 7(d)) which precluded BER measurements. At this data rate, the smooth edges of the Gaussian shape exceed the time slot available $(>6.25 \mathrm{ps})$, causing a severe cross-talk between the different channels. On the contrary, the sharper

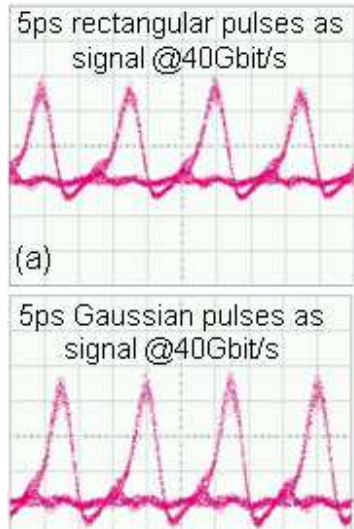

(b)

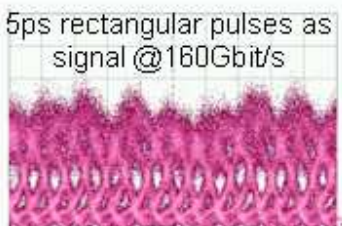

(c)

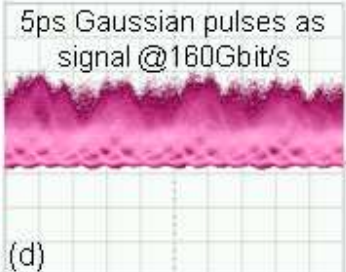

Figure 7: Eye diagrams after the Kerr switch for rectangular ( $a, c)$ and Gaussian (b,d) shapes at 40Gbit/s and $160 \mathrm{Gbit} / \mathrm{s}$ respectively.
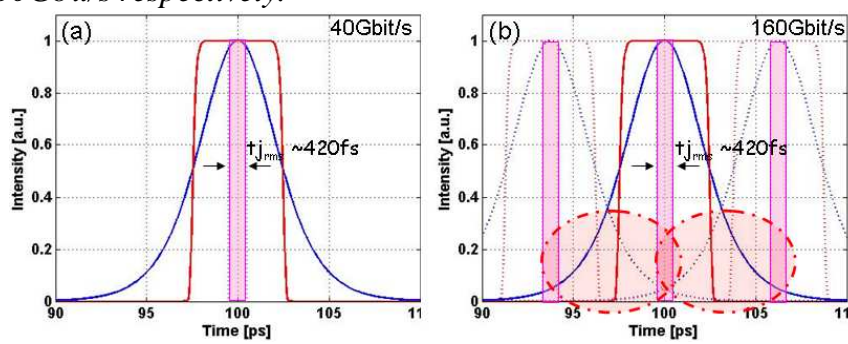

Figure 8: Sketch of the rectangular and Gaussian pulse shapes and the corresponding timing jitter of the data signal at 40Gbit/s (a) and 160Gbit/s (b).

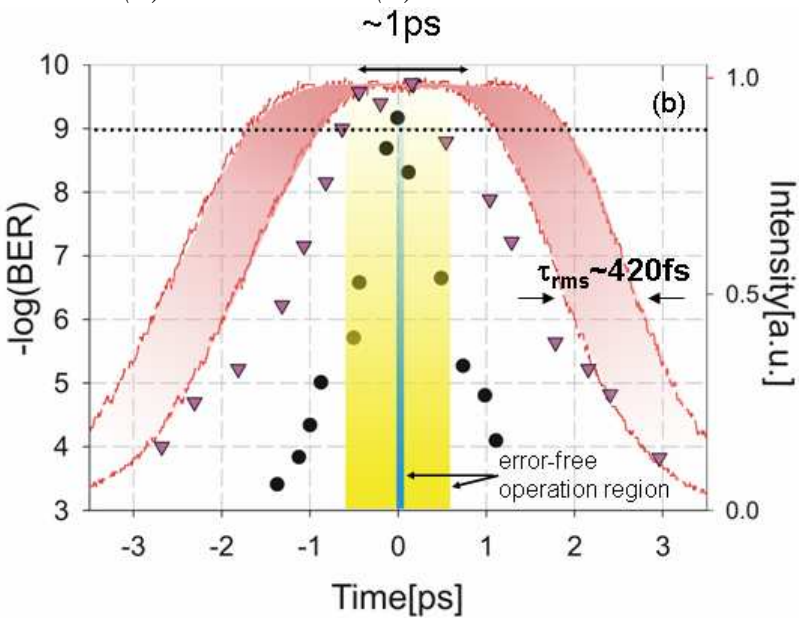

Figure 9: Measured BER at a constant received optical power $(-36 \mathrm{dBm})$ at $10 \mathrm{Gbit} / \mathrm{s}$ for flat top (triangles) and Gaussian (circles) shapes.

edges of the rectangular shape have restricted the switching window to the available time slot (see sketch in Fig. 8(b)).

Finally, we measured the BER at a constant received power of $\sim-36 \mathrm{dBm}$ as a function of the time delay between the data and the control signal and the results are shown in Fig.9. This measurement was performed at a data rate of $10 \mathrm{Gbit} / \mathrm{s}$ to avoid the need for the demultiplexer. Error free performance was readily achieved over a window of $\sim 1 \mathrm{ps}$ when the rectangular pulses are used. This is in contrast to the very narrow window provided by the $5 \mathrm{ps}$ Gaussian pulses, as also shown in Fig.9. The two shaded regions in the centre of Fig.9 indicate the conditions of error-free operation for the two different configurations, emphasizing the large improvement achieved when using rectangular pulse shaping. The same figure also shows the measured rectangular pulse shape with its associated level of timing jitter of $\tau_{\mathrm{rms}} \sim 420 \mathrm{fs}$ (shaded region), which justifies the limited extent of the effective retiming window. If the inherent timing jitter associated with the data pulse source could be eliminated, then we anticipate that error-free operation over the entire flat-top duration of the rectangular shape would have been possible.

\section{CONCLUSION}

In conclusion we have experimentally characterised a pulse retiming system based on a pulse shaping SSFBG technology. Thanks to the flexibility offered by plane-by-plane grating writing technology, we were able to generate $\sim 5$ ps rectangular pulses and demonstrate a retiming scheme at repetition rates up to $160 \mathrm{Gbit} / \mathrm{s}$. Using a flat top switching window, we clearly demonstrate a substantial performance enhancement, when compared with conventional (Gaussian) shapes, as confirmed through detailed BER measurements. Pulse shaping with SSFBGs has a range of attractive features including simple implementation, and robust, polarization insensitive operation.

\section{REFERENCES}

[1] K. Uchiyama and T. Morioka, "All-optical signal processing for $160 \mathrm{Gbit} / \mathrm{s}$ channel OTDM/WDM systems," in Proc. OFC 2001, Paper ThH2, 2001.

[2] M. Jinno, "Effects of Crosstalk and Timing Jitter on All-Optical TimeDivision Demultiplexing Using a Nonlinear Fiber Sagnac Interferometer Switch" J. Quantum Electron., vol. 30 (12), pp. 2842-2853, 1994.

[3] S. Watanabe, F. Futami, R. Okabe, Y. Takita, S. Ferber, R. Ludwig, C. Schubert, C. Schmidt-Langhorst and H. G. Weber, "160 Gb/s Optical 3RRegenerator in Fiber Transmission experiment," in Proc. of OFC 2003, Paper PD16-1, 2003.

[4] A. M. Weiner, "Femtosecond optical pulse shaping and processing," Prog. Quantum Electron., vol. 19, pp. 161-237, 1995.

[5] A. M. Weiner, J. P. Heritage and R. N. Thurston, "Synthesis of phasecoherent, picosecond optical square pulses," Opt. Lett., vol. 11, pp. 153$155,1986$.

[6] T. Kurokawa, H. Tsuda, K. Okamoto, K. Naganuma, H. Takenouchi, Y. Inoue, and M. Ishii, "Time-space-conversion optical signal procesing using 
arrayed-waveguide grating,” Electron. Lett., vol. 33 (22), pp. 1890-1891, 1997.

[7] M. Kulishov, Y. Park, J. Azana, and R. Slavik. "(Sub-) Picosecond Flattop Waveform Generation using a Single Uniform Long-Period Fiber Grating", in ECOC 2006, Paper We2.3.7, 2006.

[8] Y. Park, M. Kulishov, R. Slavik and J. Azana, "Picosecond and subpicosecond flat-top pulse generation using uniform long-period fiber gratings," Opt. Express 14 (26), 12670-12678, 2006.

[9] F.Parmigiani, L.K.Oxenlowe, M.Galili, M.Ibsen, D.Zibar, P.Petropoulos, D.J.Richardson, A.T.Clausen, P.Jeppesen, "All-optical $160 \mathrm{Gbit} / \mathrm{s} \mathrm{RZ} \mathrm{data}$ retiming system incorporating a pulse shaping fibre Bragg grating," in ECOC 2007, Berlin 16-20 Sep. 2007 (Invited).

[10] F. Parmigiani, P. Petropoulos, M. Ibsen and D. J. Richardson, "All-Optical Pulse Reshaping and Retiming Systems Incorporating Pulse Shaping Fiber Bragg Grating" IEEE J. Lightwave Technol., vol. 24 (1), pp 357-364, 2006

[11] B. J. Eggleton, P. A. Krug, L. Poladian and F. Ouellette, "Long periodic superstructure Bragg gratings in optical fibers", Electron. Lett. vol 30 (19), pp1620-1622, 1994

[12] P. Petropoulos, M. Ibsen, A. D. Ellis, and D. J. Richardson, "Rectangular Pulse Generation Based on Pulse Reshaping Using Superstructured Fiber Bragg Grating," IEEE J. Lightwave. Technol., vol. 19 (5), pp. 746-752, 2001.

[13] J H. Lee, P. C. Teh, P. Petropoulos, M. Ibsen and D. J. Richardson: "All optical modulation and demultiplexing systems with significant timing jitter tolerance through incorporation of pulse-shaping fiber Bragg gratings," IEEE Photon. Technol. Lett., vol.14 (2) pp.203-205, 2002.

[14] J. H. Lee, L. K. Oxenløwe, M. Ibsen, K. S. Berg, A. T. Clausen, D. J. Richardson, and P. Jeppesen, "Timing jitter tolerant all-optical TDM data demultiplexing at $80 \mathrm{Gbit} / \mathrm{s}$ using fiber Bragg grating based rectangular pulse switching technology," in Proc. of OFC 2003, Atlanta USA, Paper TuH7, 2003.

[15] P. C. Teh, P. Petropoulos, M. Ibsen and D. J. Richardson, "A comparative study of the performance of seven-and 63-chip optical code-division multiple-access encoders and decoders based on superstructured fiber Bragg gratings,” IEEE J. of Lightwave Technol., vol.19 (9) pp.1352-65, 2001.

[16] C. Tian, Z. Zhang, M. Ibsen, P. Petropoulos and D. J. Richardson, "Demonstration of a 16-channel code-reconfigurable OCDMA/DWDM system," in Proc. of OFC 2007, Paper OMO2, 2007.

[17] P. Petropoulos, M. Ibsen, M. N. Zervas and D. J. Richardson, "Generation of a $40 \mathrm{GHz}$ pulse stream by pulse multiplication using a sampled fiber Bragg grating," Opt. Lett., vol.25 (8) pp.521-523, 2000.

[18] P. J. Almeida, P. Petropoulos, F. Parmigiani, M. Ibsen and D. J. Richardson, "OTDM add-drop multiplexer based on timefrequency signal processing," IEEE J. of Lightwave Technol., vol.24 (7) pp.2720-2732, 2006.

[19] F. Parmigiani, P. Petropoulos, M. Ibsen and D. J. Richardson, "Pulse retiming based on XPM using parabolic pulses formed in a fiber Bragg grating," IEEE Photon. Technol. Lett., vol.18 (7) pp.829-831, 2006.

[20] F. Parmigiani, C. Finot, K. Mukasa, M. Ibsen, M. A. F. Roelens, P. Petropoulos and D. J. Richardson, "Ultra-flat SPM broadened spectra in a highly nonlinear fiber using parabolic pulses formed in a fiber Bragg grating", Optics Express, vol.14 (17) pp.7617-7622, 2006.

[21] T. T. Ng, F. Parmigiani, M. Ibsen, Z. Zhang, P. Petropoulos and D. J. Richardson, "Linear-distortion compensation using XPM with parabolic pulses," in Proc. of OFC 2007, Paper JWA58, 2007. 